\title{
Spontaneous Intracranial Hypotension Associated With Dural Sinus Thrombosis
}

\author{
-Case Report-
}

\author{
Satoru TAKEUCHI, Yoshio TAKASATO, Hiroyuki MASAOKA, Takanori \\ HAYAKAWA, Naoki OTANI, Yoshikazu YoshinO, and Hiroshi Yatsushige \\ Department of Neurosurgery, National Hospital Organization Disaster Medical Center, \\ Tachikawa, Tokyo
}

\begin{abstract}
A 32-year-old man presented with a rare case of spontaneous intracranial hypotension (SIH) associated with dural sinus thrombosis (DST) manifesting as severe postural headache which was relieved by lying down. Initial cerebrospinal fluid pressure was low. He was treated with hydration and rest under a diagnosis of SIH. Magnetic resonance (MR) imaging after 1 month showed signs of both DST and SIH. However, the patient did not demonstrate any of the classical symptoms associated with DST. The patient underwent an epidural blood patch procedure. His headache was relieved and MR imaging showed improvement of both SIH and DST. This case suggests that epidural blood patch may be effective in some cases of SIH associated with DST.
\end{abstract}

Key words: dural sinus thrombosis, spontaneous intracranial hypotension, epidural blood patch, headache

\section{Introduction}

Spontaneous intracranial hypotension (SIH) associated with dural sinus thrombosis (DST) is infrequently reported..$^{2,8,11,13)} \mathrm{SIH}$ is a rare but increasingly recognized pathological entity. The etiology of $\mathrm{SIH}$ is considered to be linked to spinal cerebrospinal fluid (CSF) leakage. The most common symptom is postural headache. ${ }^{7)}$ The magnetic resonance (MR) imaging characteristics of SIH are effacement of the basal cisterns, downward displacement of the brain, bilateral subdural effusions, diffuse pachymeningeal thickening, dilation of the dural sinuses and the spinal epidural venous plexus, and enlargement of the pituitary gland..$^{3,6,10)}$ DST is also an uncommon disease, which can manifest as numerous signs and symptoms. The typical symptoms are headache, nausea, and vomiting. DST is associated with a variety of risk factors, i.e. postpartum, thrombophilia, high dose steroid therapy, and lumbar puncture. ${ }^{1,5,9)}$

We treated a patient with SIH associated with DST, who was treated with epidural blood patch and

Received April 24, 2007; Accepted October 10, 2007 exhibited clinical and radiological improvement of both SIH and DST.

\section{Case Report}

A 32-year-old man presented with a 1-week history of occipital headache, followed by nausea. The headache worsened in the upright position for about 15 minutes and was relieved within an hour of recumbency. He was given non-steroidal antiinflammatory drugs with no benefit. Neurological examination found no abnormal signs. Computed tomography and MR imaging found no abnormalities. Serological investigations showed slightly increased white blood cell count $(8,500 / \mu \mathrm{l})$. Lumbar puncture revealed an extremely low initial CSF pressure of $5 \mathrm{cmH}_{2} \mathrm{O}$ as well as a cell count of $13 / 3 \mathrm{~mm}^{3}$ (predominantly monocytes), $55.5 \mathrm{mg} / \mathrm{dl}$ protein, 52 $\mathrm{mg} / \mathrm{dl}$ glucose, and $128 \mathrm{mEq} / \mathrm{l}$ chloride. He was treated with hydration and rest under a diagnosis of SIH.

His headache showed no remarkable change. MR imaging after 1 month showed effacement of the basal cisterns, downward displacement of the brain, bilateral subdural effusions, diffuse pachymeningeal thickening, and enlargement of the pituitary gland, consistent with the diagnosis of SIH (Fig. 1). Both 

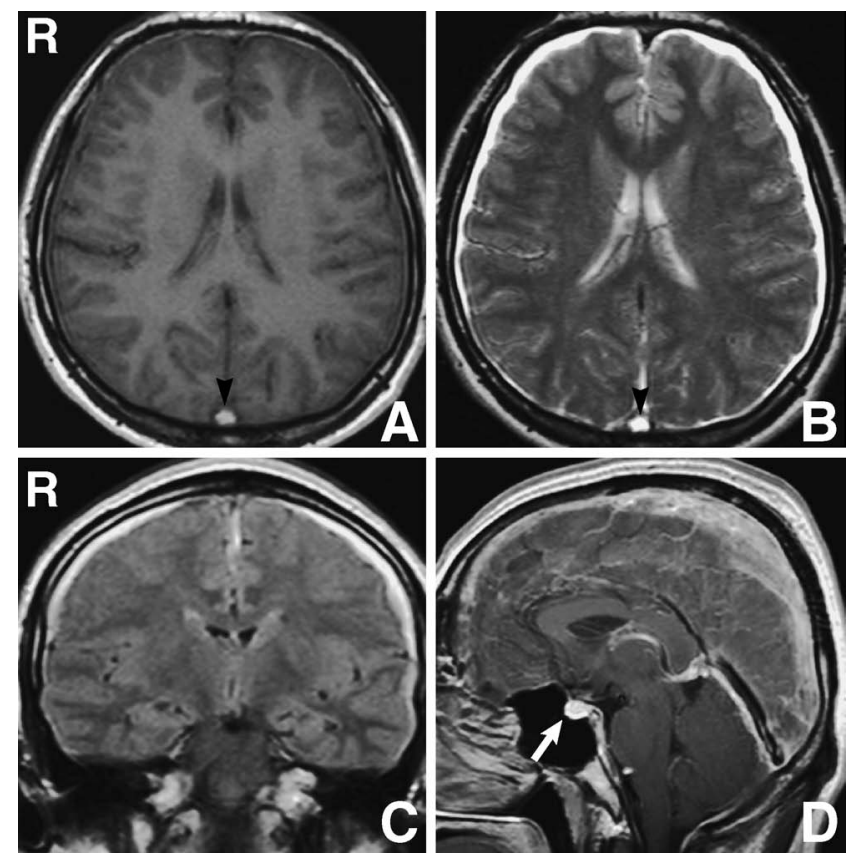

Fig. 1 Axial $\mathrm{T}_{1}$-weighted (A), axial $\mathrm{T}_{2}$-weighted (B), coronal fluid-attenuated inversion recovery (C), and sagittal $T_{1}$-weighted with gadolinium (D) magnetic resonance images 1 month after presentation showing effacement of the basal cisterns, sagging of the brain, bilateral subdural effusions, diffuse pachymeningeal thickening, and enlargement of the pituitary gland (arrow) combined with intense areas (arrowheads) within the superior sagittal sinus and the confluence of the sinuses.

$\mathrm{T}_{1}$ - and $\mathrm{T}_{2}$-weighted $\mathrm{MR}$ imaging also showed intense areas within the superior sagittal sinus and the confluence of the sinuses consistent with DST (Fig. 1). Cisternal scintigraphy showed the early appearance of urinary bladder activity and revealed no abnormal leakage at the intracranial and whole spinal subarachnoid cistern. Spinal MR imaging did not demonstrate clear evidence of the leakage site, but there was dural thickening. Angiography showed thrombosis in the superior sagittal sinus and an enlarged medullary vein which drained into the sphenoparietal sinus through the vein of Labbé (Fig. 2). Complementary screening studies for infections, and immunological, hematological, or systemic disorders did not disclose any conventional cause of DST.

He complained of worsening postural headache, consistent with SIH. Ten days later, an epidural blood patch was performed with $30 \mathrm{ml}$ of autologous blood injected at the L3-4 interspace. After 2 weeks,

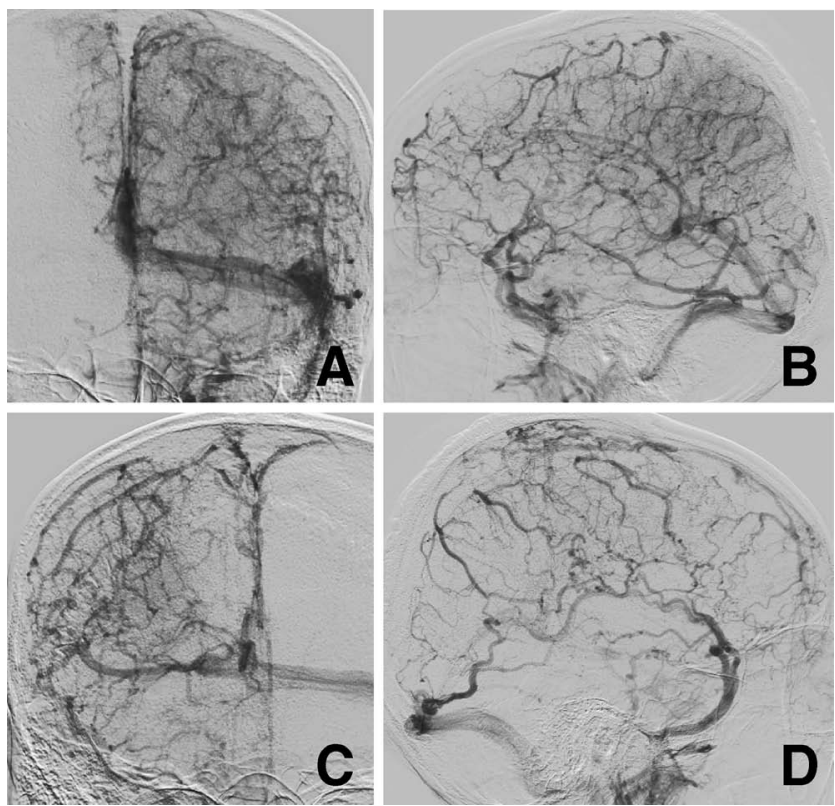

Fig. 2 Left internal carotid angiograms (anteroposterior view [A] and lateral view [B]) and right internal carotid angiograms (anteroposterior view [C] and lateral view [D]) showing no flow in the superior sagittal sinus and an enlarged medullary vein which drained into the sphenoparietal sinus through the vein of Labbé.

the headache improved and he was discharged. Follow-up MR imaging at 3 months revealed that the bilateral subdural effusions and downward displacement of the brain had disappeared. MR imaging with contrast medium detected no enhancement of the thickened dura. MR imaging and $M R$ venography also showed recanalization of the superior sagittal sinus and the confluence of the sinuses (Figs. 3 and 4). The patient remained asymptomatic at 6 months follow-up examination.

\section{Discussion}

The current hypothesis for DST in a patient with $\mathrm{SIH}$ involves engorgement of the venous system which promotes the formation of thrombosis within the dural sinuses. ${ }^{2,8,11,13)}$ DST is more common following lumbar puncture, and most cases have also had other potential risk factors for hypercoagulable states. ${ }^{1,5,9)}$ In our patient, lumbar dural puncture was performed to diagnose the SIH and may have been one of the factors causing the DST.

Patients presenting with SIH associated with DST raise difficult questions regarding the treatment of these two conditions. The first-line treatment of DST 

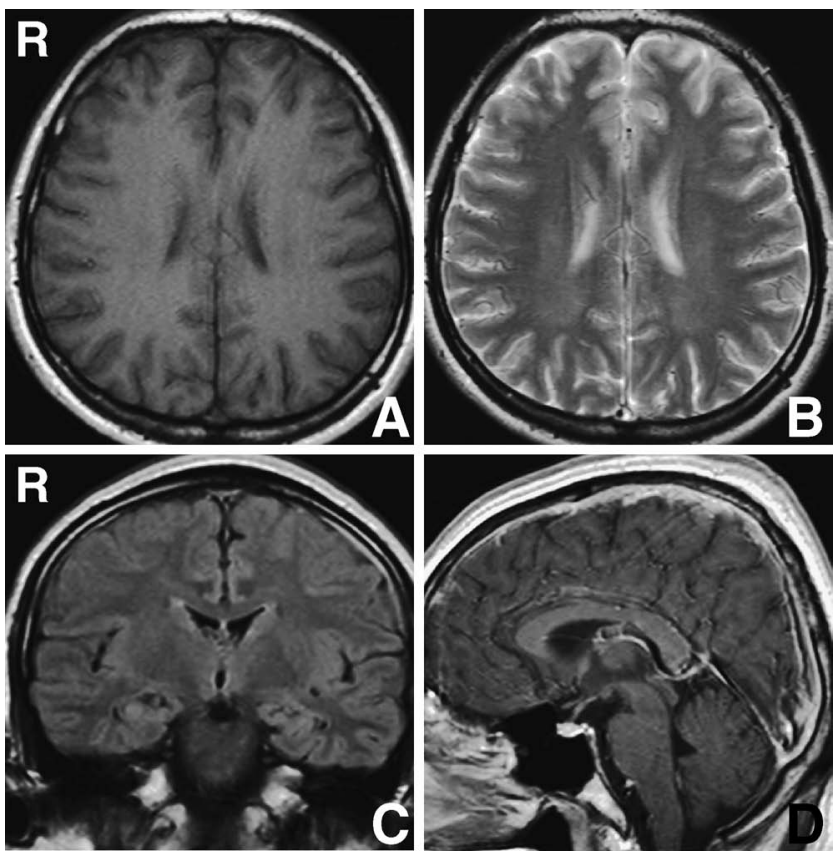

Fig. 3 Axial $T_{1}$-weighted (A), axial $T_{2}$-weighted (B), coronal fluid-attenuated inversion recovery $(C)$, and sagittal $T_{1}$-weighted with gadolinium (D) magnetic resonance images 3 months after presentation showing the disappearance of the signs of spontaneous intracranial hypotension and recanalization of the superior sagittal sinus and the confluence of the sinuses.
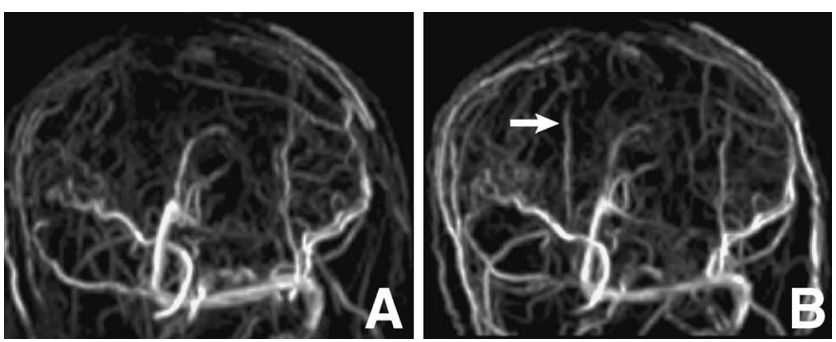

Fig. 4 Magnetic resonance venograms showing recanalization of the superior sagittal sinus and the confluence of the sinuses (arrow). A: 1 month after presentation, B: 3 months after presentation.

is heparin therapy, ${ }^{5)}$ whereas that of $\mathrm{SIH}$ is rest, hydration, or epidural blood patch. ${ }^{4,12)}$ Priority was given to the treatment of the DST in most previously reported cases, which were treated with heparin. ${ }^{2,11,13)}$ In their cases (5 patients), not only SIH but also DST were symptomatic. The DST improved, and the signs of SIH disappeared without requiring epidural blood patch. Increased intracranial pressure, which occurs in association with DST, can alleviate SIH. ${ }^{2)}$

In contrast, our patient showed severe signs of SIH, and the DST was asymptomatic. Furthermore, he had a well-developed collateral cerebral vein. Therefore, priority was given to the treatment of SIH, which we treated with an epidural blood patch. His headache then disappeared, and the MR imaging signs of both SIH and DST improved. Epidural blood patch was previously used to treat a patient with SIH and symptomatic DST who had progressive subdural hematoma. ${ }^{8)}$ The SIH directly caused the DST, and epidural blood patch indirectly cured DST. ${ }^{8)}$

The present case suggests the following. Epidural blood patch may be effective for some cases of SIH associated with DST. Assessment of whether the DST is symptomatic or asymptomatic may be important to decide whether priority is given to the treatment of SIH or DST. SIH associated with DST is rare, and CSF volume reduction is the main causative factor.

\section{Acknowledgment}

We thank Julian Cahill, M.B. for the invaluable editorial assistance in the preparation for this manuscript.

\section{References}

1) Aidi S, Chaunu MP, Biousse V, Bousser MG: Changing pattern of headache pointing to cerebral venous thrombosis after lumbar puncture and intravenous high-dose corticosteroids. Headache 39: 559-564, 1999

2) Berroir S, Grabli D, Heran F, Bakouche P, Bousser MG: Cerebral sinus venous thrombosis in two patients with spontaneous intracranial hypotension. Cerebrovasc Dis 17: 9-12, 2004

3) Chiapparini L, Ciceri E, Nappini S, Castellani MR, Mea E, Bussone G, Leone M, Savoiardo M: Headache and intracranial hypotension: neuroradiological findings. Neurol Sci 25(Suppl 3): S138-141, 2004

4) Dillon WP, Fishman RA: Some lessons learned about the diagnosis and treatment of spontaneous intracranial hypotension. AJNR Am J Neuroradiol 19: 1001-1002, 1998

5) Ferro JM, Canhao P, Stam J, Bousser MG, Barinagarrementeria F; ISCVT Investigators: Prognosis of cerebral vein and dural sinus thrombosis. Results of the International Study on Cerebral Vein and Dural Sinus Thrombosis (ISCVT). Stroke 35: 664-670, 2004

6) Fishman RA, Dillon WP: Dural enhancement and cerebral displacement secondary to intracranial 
hypotension. Neurology 43: 609-611, 1993

7) Headache Classification Subcommittee of the International Headache Society: The International Classification of Headache Disorders: 2nd edition. Cephalalgia 24 Suppl 1: 9-160, 2004

8) Kataoka H, Tanizawa E, Ueno S: Spontaneous intracranial hypotension is associated with a risk of venous sinus thrombosis and subdural hematoma. Cerebrovasc Dis 23: 315-317, 2007

9) Li JY, Lai PH, Chao KH, Peng NJ: Spontaneous intracranial hypotension: clinical and neuroimaging spectrum. Neurology 64(Suppl 1): A351, 2005

10) Miyazawa K, Shiga Y, Hasegawa T, Endoh M, Okita N, Higano S, Takahashi S, Itoyama Y: CSF hypovolemia vs intracranial hypotension in "spontaneous intracranial hypotension syndrome." Neurology 60: 941-947, 2003

11) Savoiardo $M$, Armenise S, Spagnolo $P$, De Simone T, Mandelli ML, Marcone A, Morciano G, Andreula C,
Mea E, Leone M, Chiapparini L: Dural sinus thrombosis in spontaneous intracranial hypotension: Hypotheses on possible mechanisms. J Neurol 253: 1197-1202, 2006

12) Sencakova D, Mokri B, McClelland RL: The efficacy of epidural blood patch in spontaneous CSF leaks. Neurology 57: 1921-1923, 2001

13) Sopelana D, Marcos A, Arroyo R, Gutiérrez E, Cuenca R, Vázquez AV, González JL, Egido JA: May intracranial hypotension be a cause of venous sinus thrombosis? Eur Neurol 51: 113-115, 2004

Address reprint requests to: Satoru Takeuchi, M.D., Department of Neurosurgery, National Hospital Organization Disaster Medical Center, 3256 Midori-cho, Tachikawa, Tokyo 190-0014, Japan. e-mail: satoru.t@tdmc.hosp.go.jp 